\title{
Resource Allocation for Multiuser Cooperative OFDM Networks: Who Helps Whom and How to Cooperate
}

\author{
Zhu Han, Member, IEEE, Thanongsak Himsoon, Student Member, IEEE, \\ Wipawee Pam Siriwongpairat, Member, IEEE, and K. J. Ray Liu, Fellow, IEEE
}

\begin{abstract}
Cooperative transmissions have been shown to be able to greatly improve system performance by exploring the broadcasting nature of wireless channels and cooperation among users. We focus, in this paper, on leveraging cooperation for resource allocation among users such that the network performance can be improved. Two important questions are answered-who should help whom among the distributively located users, and how many resources the users should use for cooperation to improve the performance. To answer these questions, a poweroptimization, subcarrier-allocation, and relay-selection problem is formulated over a multiuser orthogonal frequency-division multiplexing (OFDM) network, which is applicable to systems such as wireless local area networks (WLANs). In the multiuser OFDM network, cooperation among different users is conducted by assigning the subcarriers of the helping users to relay a certain part of the helped users' data, while maintaining the desired rates of both helping users and helped users by means of power control and rate adaption. This way, the bandwidth efficiency of the multiuser OFDM system with cooperation is the same as that of the noncooperative OFDM system. The formulated optimization problem is an assignment problem for subcarrier usage and corresponding bit loading as well as power control. We provide an approximate closed-form solution for a two-user two-subcarrier case. Then, a suboptimal heuristic algorithm for a multiple-user multiple-subcarrier case is proposed and implemented in the base station to solve the formulated NP-hard problem. From the simulation results, the proposed scheme achieves up to $50 \%$ overall power saving for the two-user system and $19 \%-54 \%$ overall power saving for the multiuser case with random locations, compared with the current multiuser OFDM system without cooperative diversity. The proposed scheme is also compared to a much more complicated orthogonal frequency-division multiple access (OFDMA) system.
\end{abstract}

Index Terms-Combinatorial mathematics, cooperative systems, power control, relays, resource management.

Manuscript received December 3, 2006. First published August 12, 2008 current version published May 11, 2009. Part of this work was presented at the IEEE Wireless Communications and Networking Conference, 2005. The review of this paper was coordinated by Dr. M. Dohler.

Z. Han is with the Department of Electrical and Computer Engineering, University of Houston, Houston, TX 77204 USA.

T. Himsoon and W. P. Siriwongpairat are with Meteor Communications Corporation, Kent, WA 98032 USA.

K. J. R. Liu is with the Department of Electrical and Computer Engineering and the Institute of Systems Research, University of Maryland, College Park, MD 20742 USA.

Color versions of one or more of the figures in this paper are available online at http://ieeexplore.ieee.org.

Digital Object Identifier 10.1109/TVT.2008.2004267

\section{INTRODUCTION}

$\mathbf{R}$ ESOURCE allocation, such as power control, has long been regarded as an effective way of dynamically combating channel fluctuations and reducing cochannel interference in wireless networks. The power control constantly adjusts the transmit power to maintain the received link quality, while improving the system performance. In some applications, such as wireless sensor networks in which each user is powered by batteries, optimizing the power management can greatly extend the network lifetime. Much work has been done for resource allocation for multiuser wireless networks. In [1], the authors gave an overview of radio resource management for wireless networks. In [2] and [3], a closed-loop power control framework was proposed and proved to converge to a unique optimal point. In [4], a second-order power control algorithm was proposed to improve the convergence speed. In [5], rate adaption and power control were combined to increase the system throughput. In [6] and [7], power control was combined with antenna array processing to improve the network performance. In [8], an algorithm considering time, space, and multiuser diversity was proposed for enhancing the system efficiency.

Recently, cooperative transmissions have gained much attention as an emerging transmit strategy for future wireless networks. The cooperative transmissions efficiently take advantage of the broadcasting nature of wireless networks, as well as exploit the inherent spatial and multiuser diversity. By exploring space diversity and multiuser diversity, the cooperative transmission scheme and implementation algorithms were proposed in [9], assuming full channel state information at the cooperating nodes that utilize beamforming. In [10], transmission protocols of the cooperative transmissions were classified into different approaches, and their performance was analyzed in terms of outage probabilities. The work in [11] analyzed more complicated transmitter cooperative schemes involving dirty paper coding. The authors in [12] provided rigorous analysis on symbol error rates and optimum power allocation for the multinode decode-and-forward protocol. Energy-efficient transmission was considered for broadcast networks in [13]. In [14], oversampling was combined with the intrinsic properties of orthogonal frequency-division multiplexing (OFDM) symbols, in the context of maximal ratio combining (MRC) and amplify and forward, so that this rate loss of cooperative transmissions can be overcome. In [15], the authors evaluated 
cooperative-diversity performance when the best relay is chosen according to the average SNR and the outage probability of relay selection based on the instantaneous SNR. In [16], the authors proposed a distributed relay selection scheme that requires limited network knowledge and is based on instantaneous SNRs. These ideas are also working their way into standards; for example, the IEEE 802.16 (WiMAX) standards body for future broadband wireless access has established the 802.16j Relay Task Group to incorporate cooperative relaying mechanisms into this technology.

Most of the existing cooperative transmission works concentrate on improving the one-to-one link quality, whereas most resource allocation works in the literature have not yet considered cooperation transmissions. Due to the limited number of radio resources and the increasing demand of a variety of services, it is important to consider resource allocation to fully exploit the cooperative transmission paradigm. In this paper, we consider resource allocation among multiple users to optimize the system performance by taking into consideration the cooperative transmission strategy. In multiuser wireless networks, there are many open resource allocation questions for cooperative transmissions. The most important ones are as follows: who should help whom among the distributively located users (i.e., "who helps whom" and relay selection), and how many resources (like power and subcarriers) the users should utilize for cooperation to improve the system performance ("how to cooperate"). In [17], the basic problem was formulated for a two-user case, and some preliminary heuristics were constructed. In this paper, we aim at answering these two major questions in a multiple-user case and in a more rigorous way.

To answer the questions, we consider the power-control, subcarrier-allocation, and relay-selection problem that seeks to minimize the system power over a multiuser OFDM network [18], [19], which is a key element in fourth-generation cellular networks, wireless metropolitan area networks, and wireless local area networks (WLANs). In most of the current OFDM systems, only one user transmits at a time as in a time-division duplex system, and users are scheduled for transmission over different times. The cooperation can be implemented by assigning some subcarriers of the helping users to relay parts of the helped users' data, whereas power control and rate adaption can maintain the desired rates of both helping users and helped users. By doing this, the power of the helped users is reduced greatly because some of their subcarriers have cooperative diversity and consequent power reduction that are offered by the helping users. On the other hand, the power of the helping users is increased slightly because the helping users have to not only relay the information of the helped users but also utilize the remaining subcarriers to transmit their own data by higher modulation and power. By careful design, the overall system power can be reduced. For such a shared subcarrier cooperative scheme, there is no extra stage that is purely dedicated for relays. This way, the bandwidth efficiency of the multiuser OFDM systems with cooperation is the same as that of the noncooperative OFDM systems such as the IEEE $802.11 \mathrm{a} / \mathrm{g}$ standard [20]. The optimization for the system resource allocation is performed by modifying the OFDM subcarrier assign- ment for cooperation and the corresponding bit loading as well as power control.

Furthermore, we develop a suboptimal algorithm that is implemented in the base station (BS) to solve the proposed NPhard problem. We analyze the situation where users should be helped or helping; that is, we answer the question of "who helps whom." In addition, we optimize how many OFDM subcarriers and how much power should be used for helping others; that is, we answer the question of "how to cooperate." An approximate closed-form solution for the two-user two-subcarrier case is derived. Then, a heuristic algorithm for the multiple-user multiple-subcarrier case is constructed. Performance comparison with a bound using orthogonal frequency-division multipleaccess (OFDMA) system is also studied. From the simulation results, the proposed scheme can save up to $50 \%$ of the overall transmit power for the two-user system and 19\%-54\% overall power saving for the multiple-user case with random locations, compared with the current OFDM systems without cooperative diversity.

The rest of this paper is organized as follows. In Section II, we give the multiuser OFDM system model and provide the traditional noncooperative transmission solution using the waterfilling method. In Section III, we construct the cooperative transmission over multiuser OFDM networks and formulate the cooperative optimization as an assignment problem. In Section IV, we provide algorithms to solve the problem, and simulation results are provided in Section V. Finally, Section VI concludes this paper.

\section{Multiuser OFDM System Model AND NONCOOPERATIVE SOLUTION}

We consider an uplink multiuser OFDM system. ${ }^{1}$ Suppose that there are $N$ subcarriers and $K$ users in the network. The system is time-multiplexed to serve all users, and, each time, only one user transmits. We represent $T_{i}$ as the transmission rate of the $i$ th user, and the rate is divided into $N$ subcarriers. We denote $r_{i}^{n}$ as the transmission rate of the $i$ th user at the $n$th subcarrier, and $P_{i}^{n}$ represents the corresponding transmit power. Using adaption techniques such as adaptive modulation, we have [21]

$$
r_{i}^{n}=W \log _{2}\left(1+\frac{P_{i}^{n} G_{i}^{n}}{\sigma^{2} \Lambda}\right)
$$

where $W$ is the subcarrier bandwidth, $\Lambda$ is a constant for the capacity gap, $G_{i}^{n}$ is the subcarrier gain, and $\sigma^{2}$ is the thermalnoise-plus-interference power. Without loss of generality, we assume that the noise-plus-interference power is stable, and the same for all subcarriers and all users. We also assume that the channels are constant over each power control interval.

\footnotetext{
${ }^{1}$ Since the relay can improve the performance only if it is located close to the source-destination link, we only need to consider the resource allocation within a relatively small region, i.e., within one cell. For the intercell interference that comes from the faraway sources, we consider the case in which the intercell interference either has been considered as the stable noise-plus-interference or has been solved by frequency reuse schemes.
} 
The goal of this paper is to minimize the overall power consumption under the sum rate constraint over all subcarriers for each user. If there is no cooperation among users, the overall power minimization problem is the same as independently minimizing each user's power. We define $\mathbf{P}_{i}=\left[P_{i}^{1}, \ldots, P_{i}^{N}\right]^{\prime}$ as a power assignment vector. With the bit loading in (1), the $i$ th user's power minimization problem can be expressed as

$$
\begin{aligned}
& \min _{\mathbf{P}_{i}} \sum_{n=1}^{N} P_{i}^{n} \\
& \text { s.t. } \sum_{n=1}^{N} r_{i}^{n}=T_{i} .
\end{aligned}
$$

The above constrained optimization can be solved by the traditional water-filling method [21]. By representing

$$
I_{i}^{n}=\frac{\Lambda \sigma^{2}}{G_{i}^{n}}
$$

the optimal solution of the water-filling method is given by

$$
\begin{aligned}
P_{i}^{n} & =\left(\mu_{i}-I_{i}^{n}\right)^{+} \\
r_{i}^{n} & =W \log _{2}\left(1+\frac{P_{i}^{n}}{I_{i}^{n}}\right)
\end{aligned}
$$

where $y^{+}=\max (y, 0)$, and $\mu_{i}$ is the water level that is obtained by the bisection search of the following expression:

$$
\sum_{n=1}^{N} W \log _{2}\left(1+\frac{\left(\mu_{i}-I_{i}^{n}\right)^{+}}{I_{i}^{n}}\right)=T_{i} .
$$

\section{Problem Formulation for Cooperative RESOURCE AlLOCATION}

Note that the solution in (4) is based on the assumption that all users do not cooperate with each other. Due to the broadcasting nature of wireless communication systems, not only the BS but also the other users can hear the transmitted data. If the other users can cooperate and help the transmission, cooperative diversity can be exploited, and the system performance can be improved significantly. In this section, we employ cooperative transmission over the OFDM network and then formulate the cooperative resource allocation problem.

\section{A. Cooperative Transmissions Over OFDM Networks}

In the current OFDM system such as in the IEEE $802.11 \mathrm{a} / \mathrm{g}$ standard, the media access control layer provides two different wireless access mechanisms for wireless medium sharing, namely, the distributed coordination function (DCF) and the point coordination function (PCF). The DCF achieves automatic medium sharing among users using carrier sense multiple access with collision avoidance and request to send/clear to send. The PCF is a more centralized control mechanism. In both mechanisms, time-division multiple-access (TDMA) technology is utilized for all users to share the channels. Similarly, in this paper, we assume that, at each time, only one user occupies all the bandwidth. This is also optimal for TDMA over the single-cell case due to severe interference.

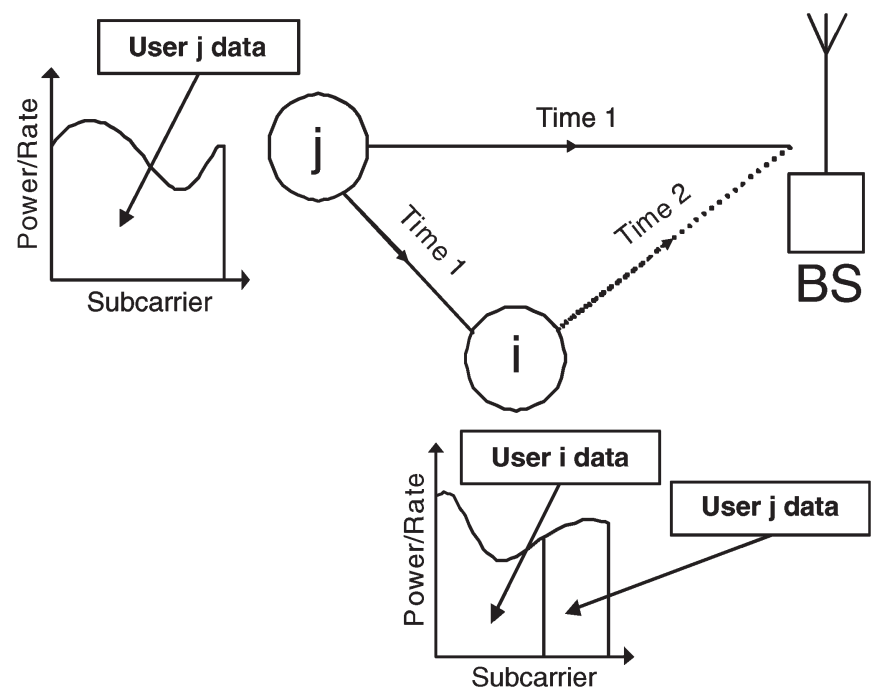

Fig. 1. OFDM cooperative transmission network.

Most of the current systems use an automatic repeat request with the acknowledgement/negative acknowledgement (ACK/NACK) mechanism to ensure the successful packet deliver. In cooperative transmission, the reason for using a relay instead of ACK/NACK is due to the wireless channel. If the source-destination wireless link is not good, there is a possibility that ACK/NACK messages may not be able to transmit successfully. Moreover, due to the bursty nature of the wireless channel, if the source-destination channel is not good now, in the immediate future, the channel might not recover yet. However, because of the broadcast nature of the wireless channel, the relay can also hear the transmission from the source to the destination. Due to spatial diversity, the relay can have a better channel condition and can help the source to transmit to the destination.

In OFDM networks, each user has the flexibility to assign the transmission over different subcarriers. This flexibility gives the possibility of cooperation among users. In this paper, we consider the cooperative OFDM system over TDMA by using this flexibility. At each time, still, only one user transmits with positive power. However, this user can select the number of subcarriers for its own data while keeping the same rate by using adaptive modulation and power control. At the same time, this user can determine the number of subcarriers for relaying parts of others' data. Notice that, unlike most of the current cooperative transmission schemes in which extra relay stages are necessary, the overall bandwidth efficiency for relays and self-transmission of the proposed system is the same as that of the current OFDM system such as the IEEE 802.11a/g standard. To fully understand the proposed scheme, an example is given as follows.

In Fig. 1, user $i$ relays user $j$ 's data to the BS. At time 1, user $j$ transmits data, whereas all other users, including the BS, can listen. In the next time period, user $i$ transmits its own data, while, at the same time, user $i$ can help transmit user $j$ 's data if user $i$ 's location is close to the BS, and the channel is good. Specifically, user $i$ can relay some parts of user $j$ 's data in some of the $N$ subcarriers to reduce user $j$ 's transmit power. In doing so, user $i$ has to transmit its own data in 


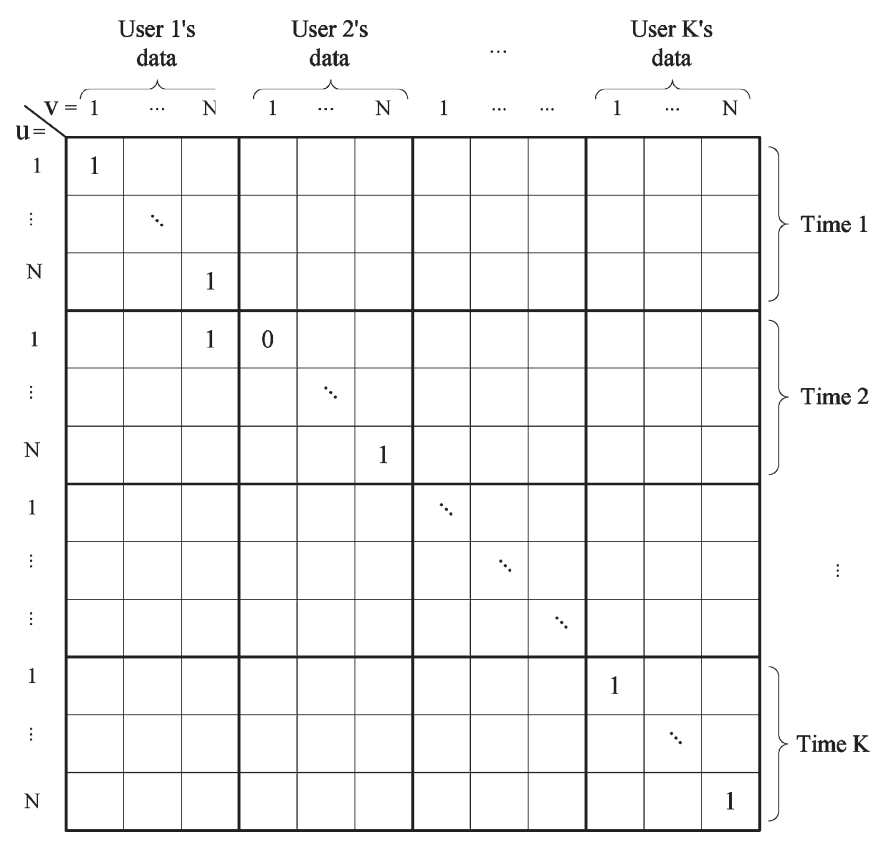

Fig. 2. Assignment matrix A example. User 2's subcarrier 1 helps user 1's subcarrier $N$.

the rest of the available subcarriers. ${ }^{2}$ Consequently, the power for user $i$ is increased to maintain its own data transmission. However, the overall system power can be reduced. From the system optimization point of view, the overall power of user $i$ and user $j$ can be minimized by selecting the proper number of subcarriers for cooperation, i.e., to answer the question of "how to cooperate." Moreover, because of the users' different locations and channel conditions, some users are more effective in helping others' transmissions. Hence, it is essential to find the optimal cooperative groups, i.e., to answer the question of "who helps whom."

We will answer the above two questions by first defining an assignment matrix $\mathbf{A}_{K N \times K N}$, given in Fig. 2, whose element $A_{u, v} \in\{0,1\}$, where $u=1, \ldots, K N$, and $v=1, \ldots, K N$. The value of $A_{u, v}$ represents the subcarrier indexes of the helping user and the helped user. For notation convenience, we denote $(i, n)=(i-1) N+n$. We use $(i, n)$ to represent the helping user $i$ at subcarrier $n$, and $\left(j, n^{\prime}\right)$ as the helped user $j$ at subcarrier $n^{\prime}$. The value of each element of $\mathbf{A}$ has the following interpretation:

1) $A_{(i, n),(i, n)}=1$ means that the $i$ th user transmits its own data at the $n$th subcarrier to the BS.

2) $A_{(i, n),\left(j, n^{\prime}\right)}=1$, for $i \neq j$, means that the $i$ th user at the $n$th subcarrier relays the data for the $j$ th user at the $n^{\prime}$ th subcarrier.

Since each subcarrier contains only the data from one user at a time, we have $\sum_{v=1}^{K N} A_{u, v}=1, \forall u=1, \ldots, K N$. Note that, in case of $\mathbf{A}=\mathbf{I}_{K N \times K N}$, the solutions of the proposed scheme are the same as those obtained from the traditional noncooperative method in Section II. We also show an example in Fig. 2 where user 2 uses its subcarrier 1 to relay the data for user 1 at the $N$ th subcarrier, i.e., $A_{(2,1),(1, N)}=1$. As shown in

\footnotetext{
${ }^{2}$ In practice, this can be implemented by adaptive modulation and by sending zero over the relaying subcarriers.
}

Fig. 2, each set of $N$ rows represents data that are transmitted at a specific time, and each set of $N$ columns represents whose data are being transmitted at that time.

\section{B. Problem Formulation for Resource Allocation Over Cooperative Transmissions}

Here, we formulate the resource allocation optimization with cooperative transmissions as an assignment problem. We define $\mathbf{P}_{K \times N}=\left[\mathbf{P}_{1}, \ldots, \mathbf{P}_{N}\right]$ as the power allocation matrix whose components are all nonnegative, and $\mathbf{G}_{K N \times K N}$ as the channel gain matrix whose elements obey the following rules.

1) $G_{\left(j, n^{\prime}\right),(i, n)}$, for $i \neq j$, denotes the channel gain from the $j$ th user at the $n^{\prime}$ th subcarrier to the $i$ th user at the $n$th subcarrier.

2) $G_{(i, n),(i, n)}$ represents the channel gain from the $i$ th user at the $n$th subcarrier to the BS.

3) To prevent $A_{(i, n),\left(i, n^{\prime}\right)}=1$, for $n \neq n^{\prime}$ (each user will not relay its own data), we define $G_{(i, n),\left(i, n^{\prime}\right)}=0, \forall n \neq n^{\prime}$ and $\forall i$.

In this paper, we use the amplified-and-forward (AF) cooperative protocol [10], which is simple to be implemented in relays and can be more mathematically tractable. Other cooperative protocols, such as decode-and-forward, can be employed in a similar way. The helping user helps the helped user by relaying the data on the selected subcarriers using AF. The receiver at the BS combines the directly received signal from the helped user and the relayed signal from the helping user using MRC. In what follows, we will derive $r_{i}^{n}$ in (1) that incorporates cooperative transmissions. Notice that helping users can select multiple subcarriers to assist the helped users. Suppose that the $i$ th user at the $n$th subcarrier helps the $j$ th user at the $n^{\prime}$ th subcarrier. We express the SNR that results from the direct transmission from the $j$ th user at the $n^{\prime}$ th subcarrier to the BS by

$$
\Gamma_{d}^{j, n^{\prime}}=\frac{A_{\left(j, n^{\prime}\right),\left(j, n^{\prime}\right)} P_{j}^{n^{\prime}} G_{\left(j, n^{\prime}\right),\left(j, n^{\prime}\right)}}{\sigma^{2}} .
$$

Here, $A_{\left(j, n^{\prime}\right),\left(j, n^{\prime}\right)}$ has a value of 0 or 1 , depending on whether user $j$ transmits its own information on subcarrier $n^{\prime}$.

Next, we consider the SNR at the BS that results from user $i$ relaying user $j$ 's data to the BS. By assuming that $X_{j, i}$ is the transmitted signal from user $j$ to user $i$, the received signal at user $i$ is given by

$$
R_{j, i}=\sqrt{P_{j}^{n^{\prime}} G_{\left(j, n^{\prime}\right)(i, n)}} X_{j, i}+\omega_{1}
$$

where $\omega_{1} \sim N\left(0, \sigma^{2}\right)$, and $\sigma^{2}$ is the noise-plus-interference variance. The noise values at different users are assumed the same. User $i$ amplifies $R_{j, i}$ and relays it to the $\mathrm{BS}$ in which the received signal is

$$
R_{i, \mathrm{BS}}=\sqrt{P_{i}^{n} G_{(i, n)(i, n)}} X_{i, \mathrm{BS}}+\omega_{2}
$$

where, without loss of generality, $\omega_{2} \sim N\left(0, \sigma^{2}\right)$ and

$$
X_{i, \mathrm{BS}}=\frac{R_{j, i}}{\left|R_{j, i}\right|}
$$


is the transmitted signal from user $i$ to the BS that is normalized to have unit energy.

Substituting (7) into (9), we can then rewrite (8) as

$$
R_{i, \mathrm{BS}}=\frac{\sqrt{P_{i}^{n} G_{(i, n)(i, n)}}\left(\sqrt{P_{j}^{n^{\prime}} G_{\left(j, n^{\prime}\right)(i, n)}} X_{j, i}+\omega_{1}\right)}{\sqrt{P_{j}^{n^{\prime}} G_{\left(j, n^{\prime}\right)(i, n)}+\sigma^{2}}}+\omega_{2} .
$$

Using (10), the relayed SNR for the $n^{\prime}$ th subcarrier of the $j$ th user, which is helped by the $n$th subcarrier of the $i$ th user, is given by

$$
\Gamma_{r}^{j, n^{\prime}}=\frac{A_{(i, n),\left(j, n^{\prime}\right)} P_{i}^{n} P_{j}^{n^{\prime}} G_{(i, n)(i, n)} G_{\left(j, n^{\prime}\right)(i, n)}}{\sigma^{2}\left(P_{i}^{n} G_{(i, n)(i, n)}+P_{j}^{n^{\prime}} G_{\left(j, n^{\prime}\right)(i, n)}+\sigma^{2}\right)} .
$$

Here, $A_{(i, n),\left(j, n^{\prime}\right)}$ has a value of 0 or 1 , depending on the relation of the helping user and the helped user. Therefore, by (6) and (11), the rate at the output of MRC is given as follows:

$$
r_{j}^{n^{\prime}}=W \log _{2}\left(1+\frac{\Gamma_{d}^{j, n^{\prime}}+\Gamma_{r}^{j, n^{\prime}}}{\Lambda}\right) .
$$

If user $i$ helps with subcarrier $n$, user $i$ transmits no information for its own data with this subcarrier, i.e., $r_{i}^{n}=0$. The overall occupied bandwidth for user $j$ 's information is, thus, $2 W$. Therefore, there is no factor of $1 / 2$ in (12). Each helping user (like user $i$ ) utilizes less bandwidth for its own transmission while using the rest of its available bandwidth (like subcarrier $n$ ) for helping others. There is no stage that is purely dedicated to the cooperative transmission. This fact leads to the same average bandwidth efficiency as the noncooperative case.

In this paper, we determine the assignment matrix $\mathbf{A}$ with combinatorial components, and the corresponding power allocation matrix $\mathbf{P}$ with nonnegative real components, for an objective of minimizing the overall power and satisfying all the constraints. The optimization problem can be formulated as

$$
\begin{aligned}
& \min _{\mathbf{A}, \mathbf{P}} \sum_{i=1}^{K} \sum_{n=1}^{N} P_{i}^{n} \\
& \text { s.t. }\left\{\begin{array}{l}
\text { transmission rate : } \sum_{n=1}^{N} r_{i}^{n}(\mathbf{A}, \mathbf{P})=T_{i}, \quad \forall i \\
\text { assignment : } \sum_{v=1}^{K N} A_{u, v}=1, \quad \forall u=1, \ldots, K N \\
\text { and } A_{u, v} \in\{0,1\}, \quad \forall u, v \\
\text { power constraint : } \sum_{n=1}^{N} P_{i}^{n} \leq P_{i}^{\max }, \quad \forall i
\end{array}\right.
\end{aligned}
$$

where $P_{i}^{\max }$ is the maximal power constraint due to the hardware limitation. Here, subscript $i$ represents users that can be helped users, helping users, or noncooperative users. Notice that A indicates both "who help whom" and the number of subcarriers for cooperation, and $\mathbf{P}$ illustrates the level of power for cooperation. From (6), (11), and (12), $r_{i}^{n}$ is a function of assignment matrix $\mathbf{A}$ and power allocation matrix $\mathbf{P}$. The optimal choice of $\mathbf{A}$ also depends on the channel conditions from the helped users to the helping users, as well as those from the helping users to the BS. In case of $\mathbf{A}=\mathbf{I}_{K N \times K N}$, the problem in (13) reduces to the noncooperative problem in (2), and the water-filling method can be used to find the optimal solution.

It is worth mentioning that the problem formulation in (13) is from a network point of view based on the assumptions that all the users will follow the protocol, and there is no greedy or malicious user. In [27], the behaviors and the incentives of greedy users are studied for cooperative transmissions.

Next, we study the maximum transmit power constraint in (13). To cope with this constraint, one common approach is adding the barrier functions, which can be written as

$$
I_{i}\left(\sum_{n=1}^{N} P_{i}^{n}, P_{i}^{\max }\right)= \begin{cases}0, & \text { if } \sum_{n=1}^{N} P_{i}^{n} \leq P_{i}^{\max } \\ \infty, & \text { otherwise. }\end{cases}
$$

We can modify the objective function in (13) as

$$
\min _{\mathbf{A}, \mathbf{P}} \sum_{i=1}^{K} \sum_{n=1}^{N} P_{i}^{n}+\sum_{i=1}^{K} I_{i}\left(\sum_{n=1}^{N} P_{i}^{n}, P_{i}^{\max }\right) .
$$

The optimization goal is equal to the original goal in (13) if each user's power is less than or equal to the maximum power limit. Otherwise, the optimization goal will achieve infinity. One good approximation for $I_{i}$ is the $\log$ function, which is widely utilized in nonlinear numerical optimization. By adding these barrier functions, the power constraint in (13) can be removed. In addition, for the single-cell case, the users that are located close to the BS have better channels and need lower transmitted power. These users can help other faraway users with higher transmitted power. The cooperation transmission reduces the helped users' power a lot and slightly increases the helping users' power. The underlying reason for this unequal power reduction and power increase is that the propagation loss factor in the wireless networks is usually greater than 1 . Since the helping users' power is usually much smaller than the helped users' power, if there exists a feasible noncooperative solution in (13), the cooperative solutions still satisfy the maximal power constraint. This fact will also be shown in the simulation results. For mathematical simplicity, we do not consider the maximal power constraint in the following analysis.

Note that the problem in (13) can be viewed as a generalized assignment problem, which is an NP-hard problem [22]. When $\mathbf{A}$ is fixed, the problem in (13) can be viewed as nonlinear continuous optimization over $\mathbf{P}$. Therefore, we divide the problem into two subproblems in Section IV. The first subproblem is finding the optimal $\mathbf{P}$ with fixed $\mathbf{A}$. Then, in the second subproblem, we try to find $\mathbf{A}$ that generates the optimal solution by using the results of the first subproblem.

\section{Proposed Resource Allocation Scheme Over COOPERATIVE TRANSMISSIONS}

In this section, we first provide an analytical approximation of optimum power allocation with fixed A for a two-user twosubcarrier case. This analysis provides some insight for the formulated problem. Second, we prove the unique optimality of (13) with fixed A. Then, based on the insight that is obtained 
from the two-user two-subcarrier case, we develop a greedy suboptimal algorithm to optimize $\mathbf{A}$ and solve the problem in (13) for the multiple-user case. Finally, a performance comparison with OFDMA for the multiuser multisubcarrier case is also provided.

\section{A. Analytical Approximation for the Two-User Two-Subcarrier System With Fixed A}

In what follows, we will show the analytical evaluation of the optimum power allocation for the cooperative transmission system with two users, as shown in Fig. 1. Since the users' locations are random and mostly asymmetric, it is effective for one user to help the other instead of both users helping each other. ${ }^{3}$ Without loss of generality, we consider the case in which user 2 helps user 1. For simplicity of the exposition, we assume that an OFDM modulator for each user utilizes two subcarriers $(N=2)$, and we consider the case that user 2 allocates subcarrier 1 to relay the data of user 1 at subcarrier $2 .{ }^{4}$ Based on the system under consideration, we know that the power of user 2 at subcarrier 2 is not used for relay transmission. Therefore, the optimum power allocation can be determined by

$$
\begin{array}{ll}
\min & P_{2}^{2} \\
\text { s.t. } & r_{2}^{2}=T_{2} .
\end{array}
$$

Thus, we have

$$
\log _{2}\left(1+\frac{G_{(2,2)(2,2)} P_{2}^{2}}{\Lambda \sigma^{2}}\right)=\frac{T_{2}}{W} .
$$

Hence, optimum power allocation $P_{2}^{2}$ is given by

$$
P_{2}^{2}=\frac{\Lambda \sigma^{2}}{G_{(2,2)(2,2)}}\left(2^{T_{2} / W}-1\right) .
$$

For the relay transmission link, $r_{2}^{1}=0$ since user 2 uses subcarrier 1 to help user 1 . Therefore, the bandwidth efficiency is the same as the noncooperative scheme. The optimum power allocation can be obtained by solving the following optimization problem:

$$
\begin{array}{ll}
\min _{\mathbf{P}} & P_{1}^{1}+P_{1}^{2}+P_{2}^{1} \\
\text { s.t. } & r_{1}^{1}+r_{1}^{2}=T_{1} .
\end{array}
$$

The Lagrange multiplier method can be applied to obtain an analytical solution. However, it is difficult, if possible, to get a

${ }^{3}$ Nevertheless, the case of two users helping each other has been studied in [23].

${ }^{4}$ This can also be generalized to the $N$-subcarrier case with a helping percentage equal to $50 \%$ by using similar techniques. closed-form solution by directly applying the Lagrange multiplier technique to (19). In the sequel, we provide an alternative approach that allows us to obtain a closed-form solution for the optimization problem in (19).

First, we use a tight approximation on the SNR expression in (11) as proved in [24]

$$
\Gamma_{r}^{1,2} \approx \frac{G_{(1,2)(2,1)} G_{(2,1)(2,1)} P_{1}^{2} P_{2}^{1}}{\sigma^{2}\left(G_{(1,2)(2,1)} P_{1}^{2}+G_{(2,1)(2,1)} P_{2}^{1}\right)} .
$$

We divide $W$ in both sides of the rate constraint in (19) and denote $\nu_{1}=T_{1} / W$. Then, we use the approximation in (20) to the constraint as that given in (21), shown at the bottom of the page, with $\nu_{1}^{1}$ corresponding to the first logarithmic term that involves only $P_{1}^{1}$, and $\nu_{1}^{2}$ relating to the second logarithmic term that contains $P_{1}^{2}$ and $P_{2}^{1}$. Both $\nu_{1}^{1}$ and $\nu_{1}^{2}$ have nonnegative values. Therefore, we can separate (19) into two subproblems, i.e.,

$$
\begin{array}{ll}
\min P_{1}^{1} & \text { s.t. } \quad \log _{2}\left(1+\frac{G_{(1,1)(1,1)} P_{1}^{1}}{\Lambda \sigma^{2}}\right)=\nu_{1}^{1}
\end{array}
$$

and

$$
\begin{aligned}
\min _{P_{1}^{2} \geq 0, P_{2}^{1} \geq 0} & P_{1}^{2}+P_{2}^{1} \\
\text { s.t. } \quad \log _{2}(1 & +\frac{G_{(1,2)(1,2)} P_{1}^{2}}{\Lambda \sigma^{2}} \\
& \left.+\frac{G_{(1,2)(2,1)} G_{(2,1)(2,1)} P_{1}^{2} P_{2}^{1}}{\Lambda \sigma^{2}\left(G_{(1,2)(2,1)} P_{1}^{2}+G_{(2,1)(2,1)} P_{2}^{1}\right)}\right)=\nu_{1}^{2} .
\end{aligned}
$$

Optimum power $P_{1}^{1}$ in (22) can be easily obtained in terms of $\nu_{1}^{1}$ as

$$
P_{1}^{1}=\frac{\Lambda \sigma^{2}}{G_{(1,1)(1,1)}}\left(2^{\nu_{1}^{1}}-1\right) .
$$

To find optimum power $P_{1}^{2}$ and $P_{2}^{1}$ in terms of $\nu_{1}^{2}$, we apply the Lagrange multiplier method to (23). After some manipulations, we find that the optimum power is the solution of a quadratic equation, i.e.,

$$
\begin{aligned}
& Y^{2} G_{(1,2)(1,2)}+G_{(1,2)(2,1)} G_{(2,1)(2,1)} Y\left(P_{2}^{1}-P_{1}^{2}\right) \\
& \quad+G_{(1,2)(2,1)} G_{(2,1)(2,1)} P_{1}^{2} P_{2}^{1}\left(G_{(1,2)(2,1)}-G_{(2,1)(2,1)}\right)=0
\end{aligned}
$$

where

$$
Y=G_{(1,2)(2,1)} P_{1}^{2}+G_{(2,1)(2,1)} P_{2}^{1} .
$$

$$
\underbrace{\log _{2}\left(1+\frac{G_{(1,1)(1,1)} P_{1}^{1}}{\Lambda \sigma^{2}}\right)}_{\nu_{1}^{1}}+\underbrace{\log _{2}\left(1+\frac{G_{(1,2)(1,2)} P_{1}^{2}}{\Lambda \sigma^{2}}+\frac{G_{(1,2)(2,1)} G_{(2,1)(2,1)} P_{1}^{2} P_{2}^{1}}{\Lambda \sigma^{2}\left(G_{(1,2)(2,1)} P_{1}^{2}+G_{(2,1)(2,1)} P_{2}^{1}\right)}\right)}_{\nu_{1}^{2}}=\nu_{1}
$$


By substituting (26) into (25), we have the quadratic form

$$
A\left(P_{1}^{2}\right)^{2}+B P_{1}^{2} P_{2}^{1}+C\left(P_{2}^{1}\right)^{2}=0
$$

where $A, B$, and $C$ are functions of channel gains only. Assuming that the channel gains are known, we can express transmit power $P_{1}^{2}$ in terms of relay power $P_{2}^{1}$ as

$$
P_{1}^{2}=\eta P_{2}^{1} .
$$

By substituting (28) into (25) and solving the quadratic equation for $\eta$, if $G_{(1,2)(2,1)} \neq G_{(2,1)(2,1)}$, we obtain (29), shown at the bottom of the page.

If $G_{(1,2)(2,1)}=G_{(2,1)(2,1)}$, then we solve for $P_{1}^{2}$ and $P_{2}^{1}$ from the optimization problem in (23) by applying the Lagrange multiplier method in a similar way. After some manipulations, we can find (30), shown at the bottom of the page, where $\Omega \triangleq 4\left(G_{(1,2)(1,2)}-G_{(1,2)(2,1)}\right)\left[\left(G_{(1,2)(1,2)}+\right.\right.$ $\left.\left.G_{(1,2)(2,1)}\right) P_{2}^{1}-G_{(1,2)(2,1)}\right]\left(P_{2}^{1}\right)^{3}$.

If $\eta$ is not a positive real number or not a number that is caused by dividing by zero in (29) and (30), because of the polynomial nature of (27), the optimal solution of (23) happens on the boundary, i.e., $P_{2}^{1}=0$. Therefore, under this condition, user 1 and user 2 do not cooperate with each other. Otherwise, if $\eta$ is a positive real number, using (28) and (29) to solve the optimization problem in (23), we can express optimum power $P_{1}^{2}$ and $P_{2}^{1}$ in terms of $\nu_{1}^{2}$ as

$$
\begin{aligned}
& P_{2}^{1}=\frac{1}{\eta \beta_{1}}\left(2^{\nu_{1}^{2}}-1\right) \\
& P_{1}^{2}=\frac{1}{\beta_{1}}\left(2^{\nu_{1}^{2}}-1\right)
\end{aligned}
$$

where

$$
\beta_{1}=\frac{G_{(1,2)(1,2)}}{\Lambda \sigma^{2}}+\frac{G_{(1,2)(2,1)} G_{(2,1)(2,1)}}{\Lambda \sigma^{2}\left(\eta G_{(1,2)(2,1)}+G_{(2,1)(2,1)}\right)} .
$$

From (24), (31), (32), and the relation $\nu_{1}^{1}=\nu_{1}-\nu_{1}^{2}$, total power $P_{1}^{1}+P_{1}^{2}+P_{2}^{1}$ that satisfies the constraint in (19) can be expressed in terms of $\nu_{1}^{2}$, i.e.,

$$
\begin{aligned}
P_{1}^{1}+P_{1}^{2}+P_{2}^{1}= & \frac{\Lambda \sigma^{2}}{G_{(1,1)(1,1)}}\left(2^{\left(\nu_{1}-\nu_{1}^{2}\right)}-1\right) \\
& +\frac{1}{\beta_{1}}\left(2^{\nu_{1}^{2}}-1\right)+\frac{1}{\eta \beta_{1}}\left(2^{\nu_{1}^{2}}-1\right) \\
\triangleq & f\left(\nu_{1}^{2}\right) .
\end{aligned}
$$

Finally, we can find the optimum value of $\nu_{1}^{2}$ by solving the unconstrained optimization problem, i.e.,

$$
\min _{\nu_{1}^{2}} f\left(\nu_{1}^{2}\right)
$$

which results in

$$
\nu_{1}^{2}=0.5 \nu_{1}+0.5 \log _{2}\left(\frac{\Lambda \sigma^{2} \eta \beta_{1}}{G_{(1,1)(1,1)}\left(\eta+\beta_{1}\right)}\right) .
$$

Consequently, $\nu_{1}^{1}$ can be found from (21) as

$$
\nu_{1}^{1}=0.5 \nu_{1}-0.5 \log _{2}\left(\frac{\Lambda \sigma^{2} \eta \beta_{1}}{G_{(1,1)(1,1)}\left(\eta+\beta_{1}\right)}\right) .
$$

Substituting the obtained $\nu_{1}^{1}$ and $\nu_{1}^{2}$ into (24), (31), and (32) gives optimum power $P_{1}^{1}, P_{1}^{2}$, and $P_{2}^{1}$, respectively.

From the above derivations, we know that, to minimize the overall power, ratio $\eta$ in (28) (i.e., the helped user's transmit power over the helping user's relay power) is only determined by the channel conditions between the two users and those to the BS. Then, for each helped user, to minimize the overall power, it balances the power allocation that is used between the subcarriers that do not get help from others and the subcarriers whose information is relayed by others. We will show by computer simulation that the above analysis with the SNR approximation in (20) provides the solutions that are very close to the optimal solutions.

\section{B. Power Minimization Optimality for the Multiple-User Multiple-Subcarrier System With Fixed A}

Here, we assume that assignment matrix $\mathbf{A}$ is known and fixed. We show the characteristics of the solution by the following theorem.

Theorem 1: For a fixed $\mathbf{A}$, as long as each cooperative subcarrier has rate $r_{j}^{n^{\prime}}$ in (12) that is larger than $W$, there is only one local optimum that is also the global optimum for (13). In other words, the corresponding SNR satisfies

$$
\Gamma_{d}^{j, n^{\prime}}+\Gamma_{r}^{j, n^{\prime}}>\Lambda
$$

Proof: All users are divided into two groups. The first group of users does not cooperate with others, i.e., $\{i$ : $A_{(i, n),\left(j, n^{\prime}\right)}=0, \forall i \neq j$ or $\left.n \neq n^{\prime}\right\}$. Therefore, the problem can be considered in the same way as the single user case,

$$
\eta=\frac{-G_{(2,1)(2,1)} G_{(1,2)(1,2)}+G_{(2,1)(2,1)} \sqrt{G_{(1,2)(2,1)}\left(G_{(2,1)(2,1)}-G_{(1,2)(1,2)}\right)+G_{(2,1)(2,1)} G_{(1,2)(1,2)}}}{G_{(1,2)(2,1)}\left(G_{(2,1)(2,1)}-G_{(1,2)(1,2)}\right)}
$$

$$
\eta=\frac{-\left(2 G_{(1,2)(1,2)} P_{2}^{1}+G_{(1,2)(2,1)}\right) P_{2}^{1}+\sqrt{\left[\left(2 G_{(1,2)(1,2)} P_{2}^{1}+G_{(1,2)(2,1)}\right) P_{2}^{1}\right]^{2}-\Omega}}{2\left(G_{(1,2)(1,2)}-G_{(1,2)(2,1)}\right)\left(P_{2}^{1}\right)^{2}}
$$


and the water-filling method can be used to find the only local optimum that is the global optimum for this kind of users.

In the second group, users cooperate with each other, i.e., $\left\{i: \exists A_{(i, n),\left(j, n^{\prime}\right)}=1, \forall i \neq j\right.$ or $\left.n \neq n^{\prime}\right\}$. For a fixed $\mathbf{A}$, the optimization problem for this group of users can be expressed as

$$
\begin{aligned}
& \min _{\mathbf{P}} \sum_{j=1}^{K} \sum_{n^{\prime}=1}^{N} P_{j}^{n^{\prime}} \\
& \text { s.t. } \quad \sum_{n^{\prime}=1}^{N} r_{j}^{n}=T_{j}, \quad \forall j .
\end{aligned}
$$

We express the constraint by the use of (12) as

$$
\prod_{n^{\prime}=1}^{N}\left(1+\frac{\Gamma_{d}^{j, n^{\prime}}+\Gamma_{r}^{j, n^{\prime}}}{\Lambda}\right)=2^{\frac{T_{j}}{W}}
$$

Since each user can be helped by at most one other user, each product for each subcarrier $n^{\prime}$ in (40) is not coupled with each other. Suppose that the cooperative rate for user $j$ and subcarrier $n^{\prime}$ is $2^{\tau}$, i.e., $\left(1+\left(\Gamma_{d}^{j, n^{\prime}}+\Gamma_{r}^{j, n^{\prime}}\right) / \Lambda\right)=2^{\tau}$. For a fixed $\mathbf{A}$ and using (6) and (11), all power components result in a quadratic form of $P_{j}^{n^{\prime}}$ because of the uncoupling fact. If the two roots of the polynomial function have opposite signs, the problem in (39) has a unique positive solution for power $\mathbf{P}$. Suppose that each cooperative subcarrier has a rate that is larger than $W$ if $\tau>1$, and the helping user $i$ helps by subcarrier $n$. From (6) and the approximation of (11), we can rewrite (40) as

$$
\begin{aligned}
G_{\left(j, n^{\prime}\right)\left(j, n^{\prime}\right)} & \left(G_{\left(j, n^{\prime}\right)\left(j, n^{\prime}\right)}+G_{(i, n)(i, n)}\right)\left(P_{j}^{n^{\prime}}\right)^{2} \\
& +C^{\prime} P_{j}^{\left(n^{\prime}\right)}+\Lambda \sigma^{2} G_{(i, n)(i, n)} P_{i}^{n}(1-\tau)=0
\end{aligned}
$$

where $C^{\prime}$ is a constant of channel gain and $P_{i}^{n}$. It is obvious that to obtain unique positive $P_{j}^{n^{\prime}}, \tau$ should be greater than 1 . As a result, the global optimum can always be achieved.

The above theorem proves that there is a unique optimal solution for the case with fixed $\mathbf{A}$ under the high rate assumption. Therefore, with fixed $\mathbf{A}$, any nonlinear or convex optimization method [25], [26] can be employed to solve (39). In the case of low rates, the optimization problem will have multiple local optima. Under this condition, there are generally two possible approaches. The first one is simulated annealing, and the other is to find good heuristic initialization.

\section{Finding Suboptimal A for the Multiple-User Multiple-Subcarrier Case}

In previous sections, we analyze the optimization problem with a fixed A. Here, we develop a suboptimal solution to find A, which represents "who helps whom" and how many subcarriers should be used for cooperation (i.e., "how to cooperate"). Because of the combinatorial nature, the problem in (39) is also an NP-hard problem [25], [26] since any element of $\mathbf{A}$ has a value of either 0 or 1 , and the search dimension of $\mathbf{A}$ is $2^{K N \times K N}$. For any specific $\mathbf{A}$, we calculate transmit power vector $\mathbf{P}(\mathbf{A})$ and then select the one that generates the minimal overall power. The problem can be formulated as

$$
\begin{aligned}
& \min _{\mathbf{A}} \sum_{i=1}^{K} \sum_{n=1}^{N} P_{i}^{n}(\mathbf{A}) \\
& \text { s.t. } \quad \sum_{v=1}^{K N} A_{u, v}=1, \quad \forall u=1, \ldots, K N \\
& \text { and } \quad A_{u, v} \in\{0,1\}, \quad \forall u, v .
\end{aligned}
$$

However, the complexity is high, particularly when a large number of subcarriers are utilized and when there are a substantial number of users in the OFDM network. This prohibits the full search method in practice.

Next, we propose a suboptimal greedy algorithm to find assignment matrix $\mathbf{A}$. The basic idea is to let the user with the least transmit power (likely close to the BS) to help the user with the most transmit power (likely far away from the BS). Consequently, the user with the least power has to increase its power to help the user with the most power in order to reduce the overall system power. Then, we determine the users' selection again and repeat the above steps. The iteration stops when no overall power can be reduced. The suboptimal greedy algorithm is implemented in the BS.

Initially, $\mathbf{A}$ is assigned as an identity matrix; that is, the initial scheme is the noncooperative scheme. Second, we sort according to users' transmit power. We select the users with the maximal and minimal transmit power as the helped user and the helping user, respectively, according to the following condition: each user has at least one subcarrier to send its own data. The above condition makes sure that the helping user's data must be transmitted. Among the $N$ subcarriers of the helping user, we make $N$ hypotheses that the $n$th subcarrier is assigned to assist the helped user, and the remaining subcarriers are unchanged. The overall power for these hypotheses is obtained by solving (39) with some numerical method. From all these hypotheses, the algorithm selects the one that maximally reduces the overall power and keeps the rest of the $N-1$ subcarriers the same in each iteration. Then, we go back to determine the helping and helped user pair again and continue the iteration. Notice that there might be more than one subcarrier of a helping user for relaying the information of the helped users. If the user with the minimal power cannot help others anymore, the random pair of the helping user and the helped user is formed to further explore the possibility that the power can be reduced. If the power cannot be reduced, this random pair of users is not applicable, and the original $\mathbf{A}$ and $\mathbf{P}$ are restored. Otherwise, the new $\mathbf{A}$ and its corresponding $\mathbf{P}$ are updated. This process stops when the power cannot be further reduced for a period of iterations. The resulting $\mathbf{A}$ is the bandwidth assignment for the helping users to the helped users, which is the answer to the questions of "who helps whom" and "how many subcarriers to cooperate." The resulting matrix $\mathbf{P}$ represents power allocation. Therefore, "how to cooperate" is also answered. Notice that, in each iteration, the sum rate for each user is kept unchanged. The detailed algorithm is shown as follows. 


\section{Multiuser Suboptimal Algorithm for A}

\author{
Initialization: $\quad \mathbf{A}=\mathbf{I}_{K N \times K N}$ and calculate \\ obtain $\mathbf{P}$.

\section{Iteration:}

1) Select user $i$ with the minimal power and user $j$ with the maximal power under the following condition:

- user $i$ has at least one subcarrier for transmitting its own data.

2) Hypotheses:

- Only the subcarrier that transmits the user's own data is eligible for the hypotheses.

- If user $i$ 's subcarrier $n$ helps user $j$ 's subcarrier $n^{\prime}$ : set $[\mathbf{A}]_{(i-1) * N+n,(i-1) * N+n}=0$ and $[\mathbf{A}]_{(i-1) * N+n,(j-1) * N+n^{\prime}}=1$.

- Solve (39) for P.

Among all hypotheses, find the maximal power reduction.

3) If all hypotheses are not effective for power reduction, random helping and helped pairs are formed to see if the power can be further reduced or not.

4) Update $\mathbf{A}$, go to step 1. End if no power reduction for a period of iterations, return $\mathbf{A}$ and $\mathbf{P}$.

Since the system power is nonincreasing in each iteration ${ }^{5}$ and is lower-bounded by the full search result, the iteration always converges. Note that the complexity for each iteration of the proposed algorithm is $O\left(N^{3}\right)$, and the algorithm is suboptimal because of the greedy local search. However, the local optimum problem is alleviated by the random disturbance in step 3 of the algorithm. Moreover, the helping users cannot be helped again in the future. As we have mentioned in the two-user case, due to the asymmetry of channel conditions for different users, it is not effective for users to help each other or form helping loop. Therefore, in the proposed algorithm, we implicitly exclude the helping loop, and users cannot be helping users and helped users at the same time.

The channel information that is required to perform the algorithm can be obtained in the following way. Since the users take turns to transmit information to the BS, the channel information from the source to the destination and from the relay to the destination can be estimated easily. When one user transmits to the BS, the possible relays of this user can estimate the channels (from sources to relays). All this information can be sent to the BS for the optimization. Since the average number of users is small, and the number of subcarriers is around 48 for WLANs, the amount of information is limited. The BS needs to send control bits to the potential helping/helped users to indicate relay selection, power optimization, and subcarrier allocation. This requires some overheard for signaling. Here, we also assume that the mobility of the users is low, so that the channel conditions are stable for sufficiently long time, and the frequency and the overhead to update the channel information are also low.

\section{Performance Comparison}

For the multiuser case, it is very difficult to obtain the analytical closed-form solution of (13) because of the nonconvexity and the integer nature of the NP-hard problem. To better understand the performance of the proposed suboptimal solution, we also compare the performance with OFDMA. Since the helped user's information is relayed by the helping user in some subcarriers, it can be viewed as the helped user occupied more bandwidth (subcarriers) by the help of the helping users. The basic idea is to view these helping subcarriers as a part of the bandwidth of the helped users. Suppose that helping user $i$ helps helped user $j$ with $N^{\prime}$ subcarriers. Therefore, the bandwidth that is occupied by the helped user is $W N\left(1+\left(N^{\prime} / N\right)\right)$, and the bandwidth for the helping user is $W N\left(1-\left(N^{\prime} / N\right)\right)$. We define each user to have the bandwidth of $\alpha_{i} N W$, where $\alpha_{i} \in(0,2]$. Note that $\alpha_{i}$ is greater than 0 since any user must have some bandwidth to transmit its own data. Since we only consider the case in which a user can only get helped by one other user, $\alpha_{i}$ is no more than 2. $\alpha_{i}$ is equal to 2 only if all the subcarriers of the helped user get helped by others. The problem formulation for the performance comparison is to minimize the overall power under the constraints of total bandwidth and individual rates, i.e.,

$$
\begin{array}{ll}
\min _{0<\alpha_{i} \leq 2} & \sum_{i=1}^{K} \alpha_{i} N P_{i} \\
\text { s.t. } \quad\left\{\begin{array}{l}
\sum_{i=1}^{K} \alpha_{i}=K \\
\alpha_{i} N W \log _{2}\left(1+\frac{P_{i} \bar{G}_{i}}{\Lambda \sigma^{2}}\right)=T_{i}
\end{array}\right.
\end{array}
$$

where $\bar{G}_{i}$ is the average channel gain ${ }^{6}$ over the bandwidth that is occupied by user $i$. The objective function is the overall transmitted power since, here, $P_{i}$ is the power per bandwidth $W$, the first constraint is the overall bandwidth, and the second constraint is the required rate. The problem in (43) is different from the problem in (13) for two reasons. First, the bandwidth assignment is relaxed to continuous functions $\alpha_{i}$ instead of discrete function A. ${ }^{7}$ Therefore, the solution to (43) is an upper bound for OFDMA. Second, the received SNR of the helped user is obtained by the assumption that all the bandwidth is directly occupied by the helped user. The purpose is to have a fair comparison from the bandwidth point of view. Moreover, this simplifies the analysis but removes the multipath diversity that is provided by cooperative transmission. Since each users' transmission can be allocated to not only the different time slots but also different frequency in (43), the multiuser, time, and frequency diversity of OFDMA can be achieved. The problem in (43) can be solved by some numerical methods [25], [26]. The solutions of (43) give us some insights on the performance comparisons of the proposed suboptimal solution, which are shown in Section V.

\footnotetext{
${ }^{6}$ Notice that flat fading is assumed for an OFDMA system to reduce the analysis complexity since OFDMA resource allocation in frequency-selective fading is still an open issue in the literature.

${ }^{7}$ This is typical relaxation in the OFDMA literature [18].

\footnotetext{
${ }^{5}$ The results from the random disturbance are applicable only when there is power reduction.
} 


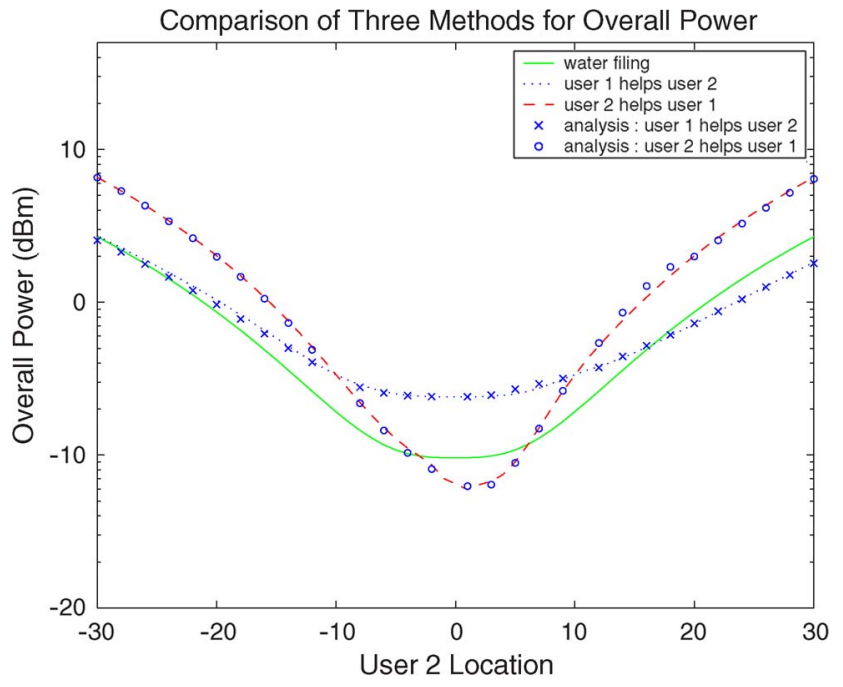

Fig. 3. Comparison of the overall power of three different schemes and analytical results.

\section{Simulation Results And Analysis}

We perform computer simulations for multiuser OFDM systems to evaluate the system performance and answer the questions like "who helps whom" and "how to cooperate." In what follows, the simulation results for the two-user system are presented in the first part, and those for the multiuser system are shown in the second part.

\section{A. Two-User System}

We set the simulations of the first part as follows. There are a total of $K=2$ users in the OFDM network. A BS is located at coordinate $(0,0)$, user 1 is fixed at coordinate $(10 \mathrm{~m}, 0)$, and user 2 is randomly located within the range of $[-30 \mathrm{~m}, 30 \mathrm{~m}]$ in both the $x$ - and $y$-axes. The propagation loss factor is set to 3 . The noise-plus-interference level is $\sigma^{2}=-60 \mathrm{dBm}$, and we select the capacity gap as $\Lambda=1$. An OFDM modulator for each user utilizes $N=32$ subcarriers, and each subcarrier occupies bandwidth of $W=1$. Without loss of generality, we assume flat fading in simulations, so that the results, such as regions, can be clearly related to distances and can be compared with the OFDMA results obtained from Section IV-D. The system under frequency-selective fading can be studied in a similar way. The performance can be further improved by exploring frequency diversity.

In Fig. 3, we show a comparison of the overall power in decibels of the water-filling scheme, the user 1 helps user 2 (1-H-2) scheme, the user 2 helps user $1(2-\mathrm{H}-1)$ scheme, and the analyzed results from Section IV-B. In this simulation, user 2 moves from location $(-30 \mathrm{~m}, 0)$ to $(30 \mathrm{~m}, 0)$. The transmission rate for each user is $T_{i}=2 N W$, and half of the subcarriers are used for helping the other. We observe that when user 2 is located close to the BS, the 2-H-1 scheme can reduce the overall power up to $50 \%$. The reason is that user 1 can use user 2 as a relay node to transmit user 1's data such that user 1's power can be reduced. Since user 2 is close to the BS, even with only half of the subcarriers to carry its own data, the increase in power for user 2 is still smaller than the power reduction for

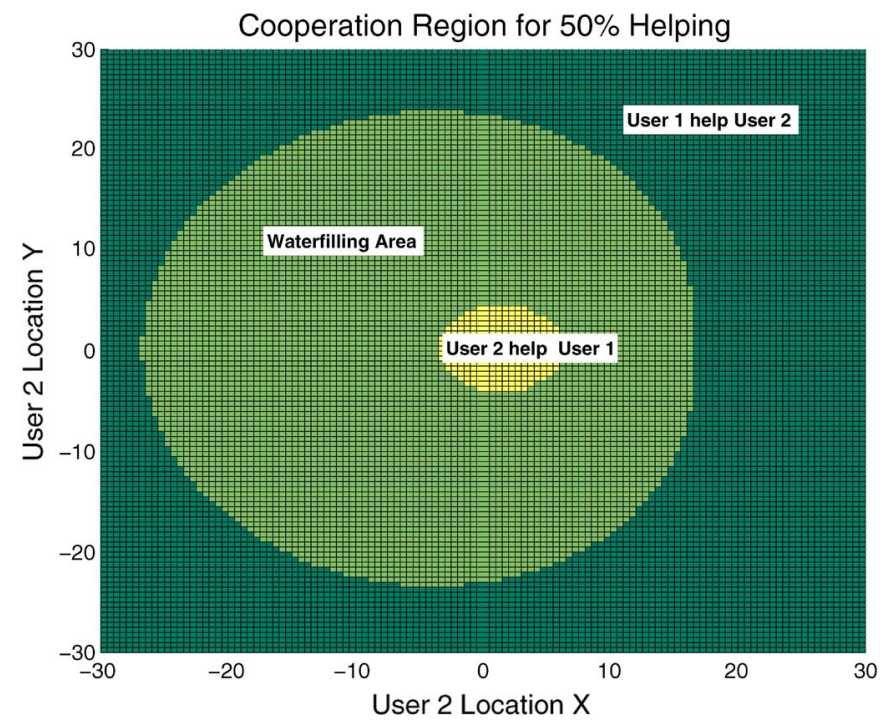

Fig. 4. Cooperative region for a two-user system with user 1 located at $(10 \mathrm{~m}, 0 \mathrm{~m})$

user 1. On the other hand, when user 2 is located far away from the BS, even in the opposite direction to user 1 such as $(-30 \mathrm{~m}, 0)$, the $1-\mathrm{H}-2$ scheme can reduce the overall transmit power. This can be explained by the same reason as above. In the extreme case, when user 2 is located very far away from the BS compared with the location of user 1 to the BS, user 1 and the BS can be considered as multiple sinks for user 2's signal. This provides the so-called "virtual multiple antenna diversity." In addition, the analytical results that are obtained by derivation closely match the optimal results by the numerical algorithm.

In Fig. 4, we show the region where different schemes should be applied based on user 2's location. With the same simulation setup as in the previous case, we can see that when user 2's location is close to the BS or lies in between user 1 and the BS, the 2-H-1 scheme is preferred. When user 2 is located far away from the BS, the 1-H-2 scheme produces minimal overall power. When user 2's location is in between the above two cases, the water-filling scheme is the optimum choice. This figure presents the answers to the question of "who helps whom." It is worth mentioning that both $x$ - and $y$-axes represent users' channel conditions by using locations, so that the answer of "who helps whom" can be illustrated clearly. If channel effects such as shadowing and fading are considered, both $x$ - and $y$-axes can be channel conditions and are not related to the distances.

In Fig. 5, we answer the question of how the users should cooperate with each other. The simulation setup is the same as in the previous case except that the number of helped subcarriers is not fixed. Instead, we find the optimal percentage of subcarriers that are used for helping others. We show the helping percentage as a function of different user 2's locations. For pure notation purposes, when the $2-\mathrm{H}-1$ scheme is used, the percentage is illustrated to be positive; when the waterfilling scheme is chosen, the percentage is zero; and when the 1-H-2 scheme is applied, the percentage is shown to be negative. By doing this, with the same percentages, we can distinguish who helps whom. From Fig. 5, the closer user 2 


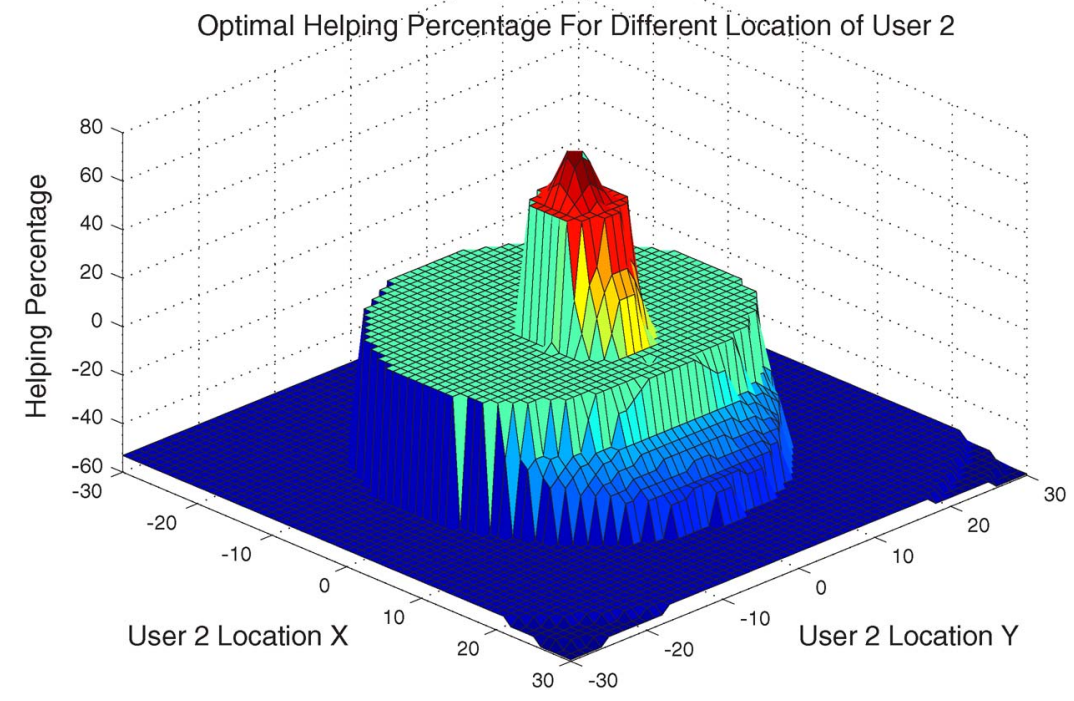

Fig. 5. Cooperative percentage for a two-user system.

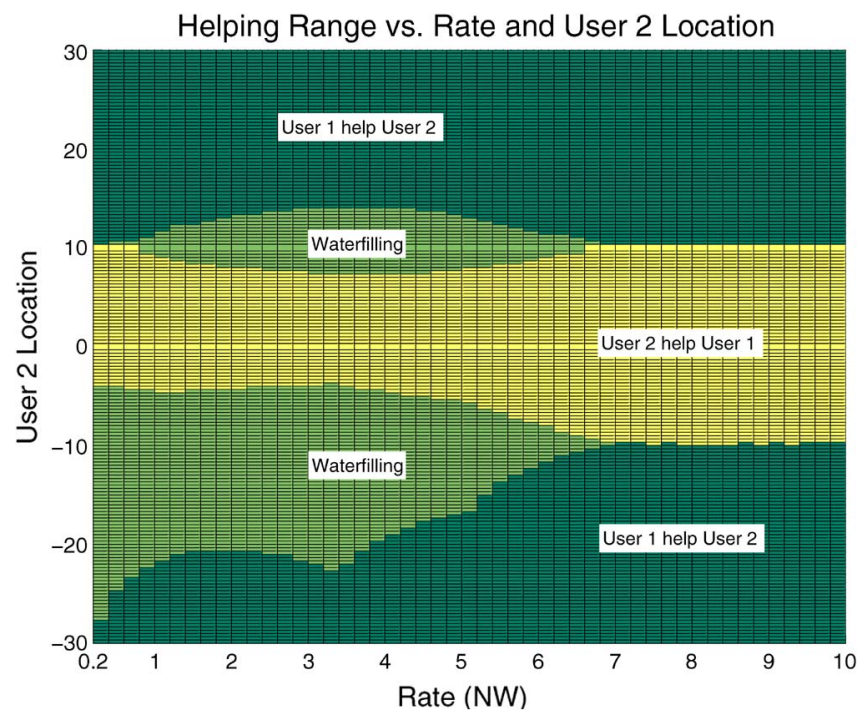

Fig. 6. Cooperative region versus the user's rate.

is to the BS, the larger the percentage user 2 will help user 1. This observation follows the fact that user 2's own transmission virtually costs nothing in terms of power usage. On the other hand, when user 2 is far away from the BS, user 1 increasingly helps user 2. Therefore, the figure gives us insight as to how the level of cooperation and partner assignments affect the system performance.

Figs. 6 and 7 show the effects of a user's transmission rate $T_{i}$ on who should help whom and how users should cooperate. We modify $T_{i}$ from $0.5 \mathrm{NW}$ to $10 \mathrm{NW}$ for all users. User 2 is located from $(-30 \mathrm{~m}, 0)$ to $(30 \mathrm{~m}, 0)$. As we can see from Fig. 6, the helping regions are changed with the increase in the transmission rate. The water-filling regions increase to the maxima around the rate equal to $3.4 N \mathrm{~W}$, and then the water-filling regions are shrunk until all regions are occupied by $1-\mathrm{H}-2$ or $2-\mathrm{H}-1$ regions. This is because when the power exponentially grows with the increase in the rate, the helping with little percentage can reduce a lot of power in the proposed
OFDM cooperative network. In Fig. 7, however, we observe that the helping percentages are reduced as the users' rates keep increasing. This is because the users need more subcarriers to send their own data; therefore, the number of subcarriers to help others is reduced. Note also from the figure that when the rate is high, the original water-filling area becomes the $1-\mathrm{H}-2$ or $2-\mathrm{H}-1$ area. However, the percentage of helping is very small.

\section{B. Multiuser System}

Here, we consider the performance of the proposed scheme for the multiuser scenario. In the simulations, all distributed users are located within a circle of radius $50 \mathrm{~m}$. The BS is located at the center of the circle, i.e., $(0 \mathrm{~m}, 0 \mathrm{~m})$, and the closest distance from a user to the BS is $1 \mathrm{~m}$. The other settings are the same as those for the two-user case.

In Fig. 8, we show the simulation results for a four-user case. Over different random locations, we select a typical snapshot of users $1,2,3$, and 4 with locations $(3.2331 \mathrm{~m}$, $7.2071 \mathrm{~m}),(1.7768 \mathrm{~m},-7.8635 \mathrm{~m}),(35.7636 \mathrm{~m}, 5.8430 \mathrm{~m})$, and $(-32.7265 \mathrm{~m}, 21.5281 \mathrm{~m})$, respectively. We show the power changes in the proposed cooperative scheme versus the iteration number. Because user 1 and user 2 are close to the BS, they help the transmission of user 3 and user 4 to reduce their power. The subcarriers of user 1 and user 2 are assigned to help user 3 and user 4 . Consequently, the remaining numbers of subcarriers for user 1 and user 2 are reduced, so that their transmit power is increased. However, the overall system power is reduced. Notice that after iteration 37, user 4 still has the largest power; however, no user can further help him/her since helping user 4 will increase the power of the helping users more than the power reduction of user 4. From iteration 50, user 2 tries to help user 3, which has the second largest power. This is due to the third step of the algorithm, in which the random pair of users is formed.

In Fig. 9, we show the system overall power in decibels versus the number of users with different rate requirements. We can see that the proposed cooperative scheme can save up to 


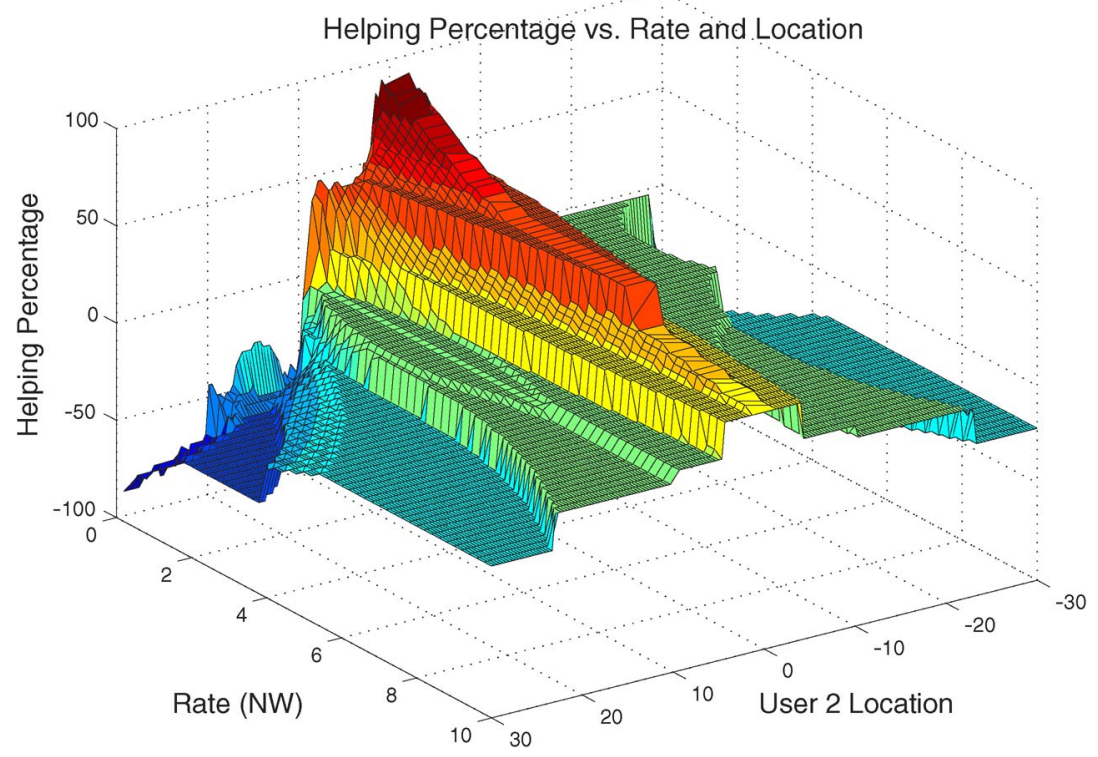

Fig. 7. Helping percentage versus the user's rate.

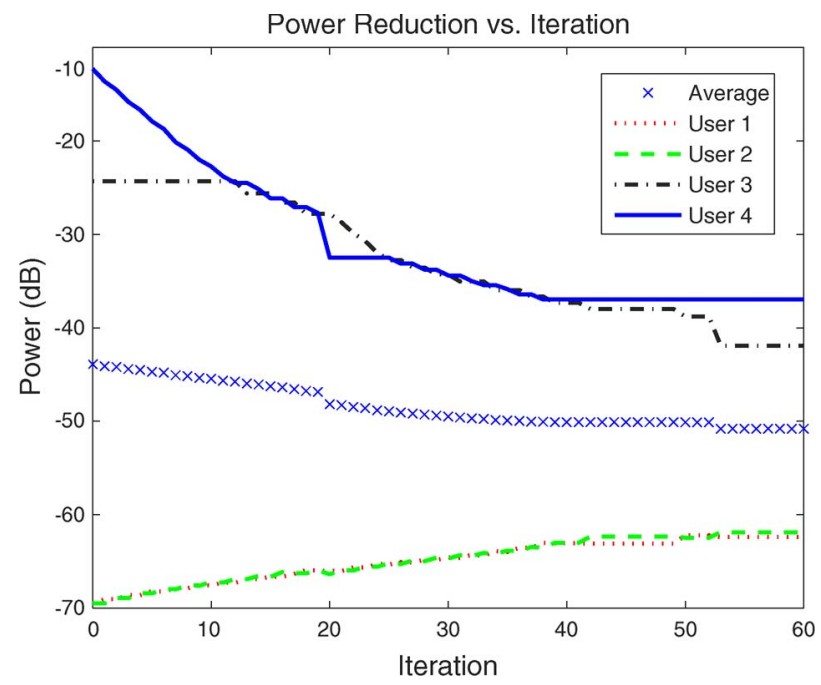

Fig. 8. Power convergence.

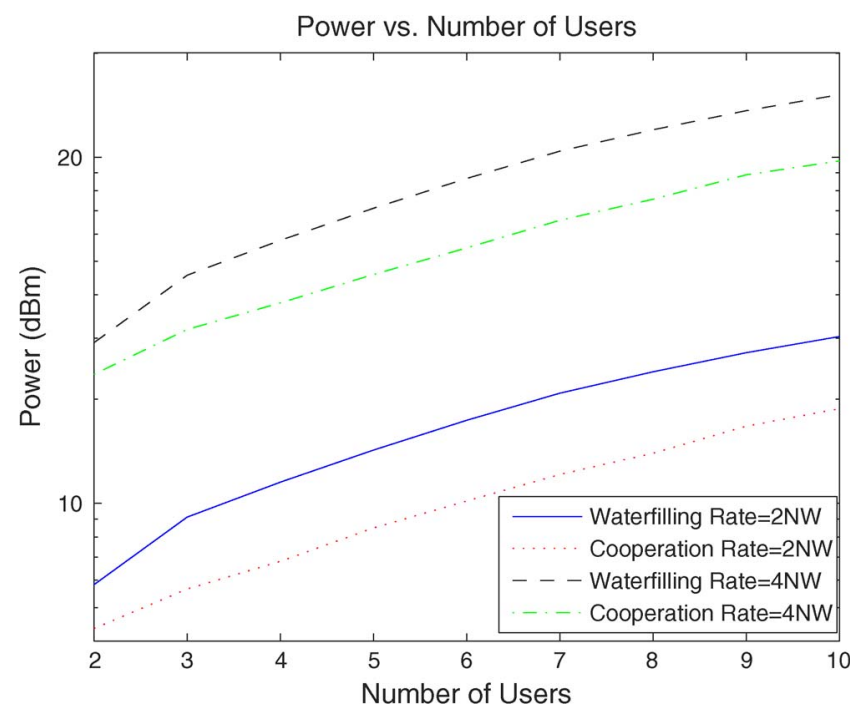

Fig. 9. Overall power versus the number of users.

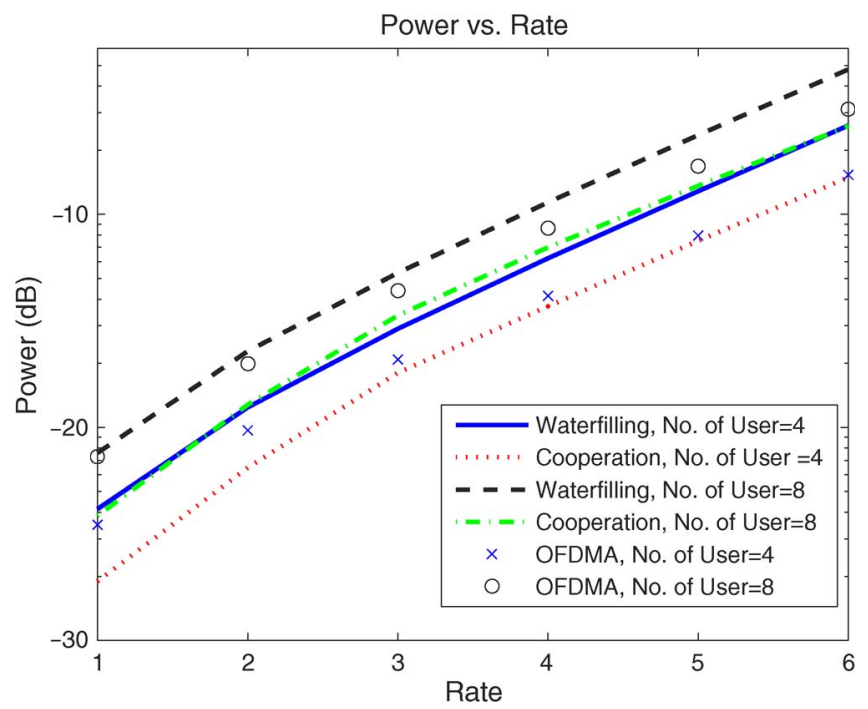

Fig. 10. Overall power versus each user's rate in the unit of $N W$.

$19 \%$ of overall power for a rate equal to $2 N W$ and $42 \%$ for a rate equal to $4 N W$, respectively. The power reduction increases when the number of users is large, which is due to the increase in the choices for cooperation. In the simulation, we find some occasions where the users are located in the areas where the water-filling scheme will produce the minimal overall power. Under this occasion, the proposed cooperative scheme has the same performance as the noncooperative scheme.

In Fig. 10, we show the overall system power versus the rate requirements with four-user and eight-user cases. Obviously, the overall power increases almost exponentially with the rate requirement. This corresponds to the relation between the rate and the power given in (1) and (12). The proposed cooperative scheme can reduce $37 \%-54 \%$ of the overall power under the four-user and eight-user conditions. The proposed cooperative scheme can reduce more power when the rate is high. This is because the power reduction is large if the user is helped by others when the rate is higher, although the percentages 
TABLE I

EFFect of Shadow Fading on Power SaVing (Percentage of Power Reduction Using CoOperation OVER the POWER OF NONCOOPERATION)

\begin{tabular}{|c|c|c|}
\hline Shadow Fading Standard Deviation & Rate=2NW & Rate=4NW \\
\hline $1 \mathrm{~dB}$ & $23.5 \%$ & $37.3 \%$ \\
\hline $3 \mathrm{~dB}$ & $41.0 \%$ & $53.5 \%$ \\
\hline $5 \mathrm{~dB}$ & $59.5 \%$ & $78.9 \%$ \\
\hline
\end{tabular}

of subcarriers that other users would like to help are reduced according to Fig. 7.

In Table I, we investigate the shadow fading effect on the proposed scheme. Specifically, we study the lognormal shadow fading model with different variances in decibels. As shown in the table, the proposed scheme can achieve more power saving (defined as the percentage of power reduction using cooperation over the power of noncooperation using water filling) when shadow fading is more severe. The intuitive reason is that the variance for the channel gains between different users increases. As a result, the proposed scheme can have more opportunities to improve the system performance.

In Fig. 10, we also show the performance comparisons using (43), which is an upper bound for the OFDMA system. The proposed suboptimal scheme has better performance in the lower rate region and similar results in the higher rate region, compared with the OFDMA upper bound. Notice that, for our proposed scheme, each time, only one user occupies all the frequency; that is, the system is still a time-division multiplex system. For the OFDMA system, at each time, more than one user is transmitting by using different subcarriers. The synchronization of different users' frequency is necessary to prevent intersubcarrier interference. This synchronization can pose significant implementation challenges to the system design. By using the proposed cooperative OFDM system, the complexity of synchronization in the OFDMA system is avoided.

\section{CONCLUSION}

We have proposed, in this paper, the cooperative resource allocation over multiuser OFDM networks by exploring the broadcasting nature of wireless channels and, consequently, the possible cooperation among users to improve the system performance. We have targeted the answer to two resource allocation questions in cooperative transmissions: "who helps whom" and "how to cooperate." To answer these questions, we have formulated an assignment NP-hard problem and proposed algorithms to solve it. The proposed scheme not only can find the pairs of a helping user and a helped user to cooperate but also can obtain how many subcarriers should be used for cooperation. Moreover, an analytical closed-form solution for the two-user case and a numerical performance comparison with OFDMA have been provided, which allow us to get some insight on the performance of the proposed algorithm. From the simulation results, we have known in which range the cooperative scheme and the noncooperative scheme should be applied, and how many resources should be used for cooperation. The proposed scheme can significantly save the overall transmit power for the two-user case and the multiple-user case.

\section{REFERENCES}

[1] J. Zander and S. L. Kim, Radio Resource Management for Wireless Networks. Norwood, MA: Artech House, 2001.

[2] R. Yates, "A framework for uplink power control in cellular radio systems," IEEE J. Sel. Areas Commun., vol. 13, no. 7, pp. 1341-1347, Sep. 1995.

[3] G. J. Foschini and Z. Miljanic, "A simple distributed autonomous power control algorithm and its convergence," IEEE Trans. Veh. Technol., vol. 42, no. 4, pp. 641-646, Nov. 1993.

[4] R. Jantti and S. L. Kim, "Second-order power control with asymptotically fast convergence," IEEE J. Sel. Areas Commun.: Wireless Commun. Series, vol. 18, no. 3, pp. 447-457, Mar. 2000.

[5] X. Qiu and K. Chawla, "On the performance of adaptive modulation in cellular systems," IEEE Trans. Commun., vol. 47, no. 6, pp. 884-895, Jun. 1999.

[6] F. Rashid-Farrokhi, L. Tassiulas, and K. J. R. Liu, "Joint optimal power control and beamforming in wireless networks using antenna arrays," IEEE Trans. Commun., vol. 46, no. 10, pp. 1313-1324, Oct. 1998.

[7] F. Rashid-Farrokhi, K. J. R. Liu, and L. Tassiulas, "Transmit beamforming and power control for cellular wireless systems," IEEE J. Sel. Areas Commun., vol. 16, no. 8, pp. 1437-1450, Oct. 1998.

[8] Z. Han and K. J. R. Liu, "Power minimization under throughput management over wireless networks with antenna diversity," IEEE Trans. Wireless Commun., vol. 3, no. 6, pp. 2170-2181, Nov. 2004.

[9] A. Sendonaris, E. Erkip, and B. Aazhang, "User cooperation diversity—Part I: System description," IEEE Trans. Commun., vol. 51, no. 11, pp. 1927-1938, Nov. 2003.

[10] J. N. Laneman, D. N. C. Tse, and G. W. Wornell, "Cooperative diversity in wireless networks: Efficient protocols and outage behavior," IEEE Trans. Inf. Theory, vol. 50, no. 12, pp. 3062-3080, Dec. 2004.

[11] A. Host-Madsen, "A new achievable rate for cooperative diversity based on generalized writing on dirty paper," in Proc. IEEE Int. Symp. Inf. Theory, Yokohama, Japan, Jun. 2003, p. 317.

[12] A. K. Sadek, W. Su, and K. J. R. Liu, "Multi-node cooperative communications in wireless networks," IEEE Trans. Signal Processing, vol. 55, no. 1, pp. 341-355, Jan. 2007.

[13] I. Maric and R. D. Yates, "Cooperative multihop broadcast for wireless networks," IEEE J. Sel. Areas Commun., vol. 22, no. 6, pp. 1080-1088, Aug. 2004.

[14] A. Bletsas and A. Lippman, "Efficient collaborative (viral) communication in OFDM based WLANs," in Proc. ISART, Boulder, CO, Mar. 2003, pp. 27-32.

[15] J. Luo, R. S. Blum, L. J. Greenstein, L. J. Cimini, and A. M. Haimovich, "New approaches for cooperative use of multiple antennas in ad hoc wireless networks," in Proc. IEEE Veh. Technol. Conf.-Fall, Los Angeles, CA, Sep. 2004, pp. 2769-2773.

[16] A. Bletsas, A. Lippman, and D. P. Reed, "A simple distributed method for relay selection in cooperative diversity wireless networks, based on reciprocity and channel measurements," in Proc. IEEE Veh. Technol. Conf.-Spring, Stockholm, Sweden, May 2005, pp. 1484-1488.

[17] Z. Han, T. Himsoon, W. Siriwongpairat, and K. J. R. Liu, "Energyefficient cooperative transmission over multiuser OFDM networks: Who helps whom and how to cooperate," in Proc. IEEE Wireless Commun. Netw. Conf., New Orleans, LA, Mar. 2005, vol. 2, pp. 1030-1035.

[18] C. Y. Wong, R. S. Cheng, K. B. Letaief, and R. D. Murch, "Multiuser OFDM with adaptive subcarrier, bit, and power allocation," IEEE J. Sel. Areas Commun., vol. 17, no. 10, pp. 1747-1758, Oct. 1999.

[19] W. Yu and J. M. Cioffi, "FDMA capacity of Gaussian multiple-access channels with ISI," IEEE Trans. Commun., vol. 50, no. 1, pp. 102-111, Jan. 2002.

[20] Wireless LAN Medium Access Control (MAC) and Physical Layer (PHY) Specifications, Aug. 1999. ANSI/IEEE Std. 802.11.

[21] T. M. Cover and J. A. Thomas, Elements of Information Theory. New York: Wiley-Interscience, 1991.

[22] S. Martello and P. Toth, Knapsack Problems: Algorithms and Computer Implementations. New York: Wiley, 1990.

[23] T. Himsoon, W. P. Siriwongpairat, Z. Han, and K. J. R. Liu, "Lifetime maximization via cooperative nodes and relay deployment in wireless networks," IEEE J. Sel. Areas Commun., Special Issue on Cooperative Communications and Networking, vol. 25, no. 2, pp. 306-317, Feb. 2007.

[24] M. O. Hasna and M. S. Alouini, "Performance analysis of two-hop relayed transmissions over Rayleigh fading channels," in Proc. IEEE VTC, Sep. 2002, vol. 4, pp. 1992-1996.

[25] S. Boyd and L. Vandenberghe, Convex Optimization. Cambridge, U.K.: Cambridge Univ. Press, 2006. 
[26] Z. Han and K. J. R. Liu, Resource Allocation for Wireless Networks: Basics, Techniques, and Applications. Cambridge, U.K.: Cambridge Univ. Press, 2008.

[27] B. Wang, Z. Han, and K. J. R. Liu, "Stackelberg game for distributed resource allocation over multiuser cooperative communication networks," in Proc. IEEE Global Telecommun. Conf., San Francisco, CA, Nov. 2006, pp. $1-5$.

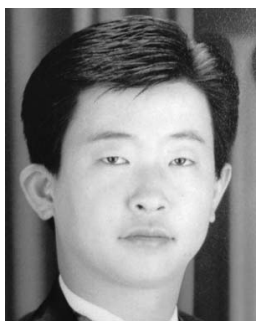

Zhu Han (S'01-M'04) received the B.S. degree in electronic engineering from Tsinghua University, Beijing, China, in 1997 and the M.S. and Ph.D. degrees in electrical engineering from the University of Maryland, College Park, in 1999 and 2003, respectively.

From 2000 to 2002, he was an R\&D Engineer with JDS Uniphase Corporation, Germantown, MD. From 2002 to 2003, he was a Graduate Research Assistant and, from 2003 to 2006, a Research Associate with the University of Maryland. From 2006 to 2008, he was an Assistant Professor with Boise State University, Boise, ID. During June-August 2006, he was a Visiting Scholar with Princeton University, Princeton, NJ; during May-August 2007, he was a Visiting Professor with Stanford University, Stanford, CA; and during May-August 2008, he was a Visiting Professor with the University of Oslo, Oslo, Norway, and Supelec, Paris, France. He is currently an Assistant Professor with the Department of Electrical and Computer Engineering, University of Houston, Houston, TX. $\mathrm{He}$ is the Guest Editor for the Special Issue on Fairness of Radio Resource Management Techniques in Wireless Networks of the EURASIP Journal on Wireless Communications and Networking and Special Issue on Game Theory of the EURASIP Journal on Advances in Signal Processing. His research interests include wireless resource allocation and management, wireless communications and networking, game theory, wireless multimedia, and security.

Dr. Han is the MAC Symposium Vice Chair of the 2008 IEEE Wireless Communications and Networking Conference. He is a Member of the Technical Programming Committee for the IEEE International Conference on Communications, the IEEE Vehicular Technology Conference, the IEEE Consumer Communications and Networking Conference, the IEEE Wireless Communications and Networking Conference, and the IEEE Globe Communication Conference.

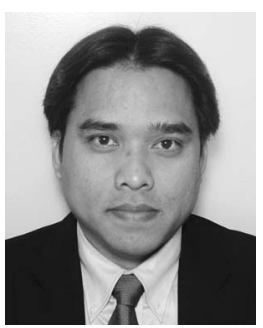

Thanongsak Himsoon (S'03) received the B.S. degree in electrical engineering from the Chulalongkorn University, Bangkok, Thailand, in 1995 and the M.S. and Ph.D. degrees in electrical engineering from the University of Maryland, College Park, in 2001 and 2006, respectively.

$\mathrm{He}$ is currently a Communications Systems Design Engineer with Meteor Communications Corporation, Kent, WA, working on algorithm and system design for wireless communications. His research interests include signal processing, wireless communications, cooperative communications, and wireless sensor networks, with particular focus on differential modulation systems. His research contributions encompass differential space-time coding and modulation for multipleinput-multiple-output (MIMO) systems, MIMO-orthogonal frequency-division multiplexing, ultrawideband systems, and cooperative communications.

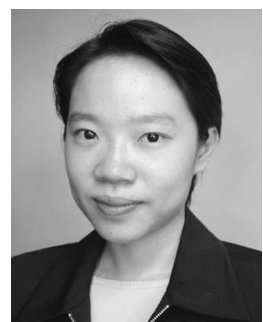

Wipawee Pam Siriwongpairat (S'03-M'06) received the B.S. degree in electrical engineering from the Chulalongkorn University, Bangkok, Thailand, in 1999 and the M.S. and Ph.D. degrees in electrical engineering from the University of Maryland, College Park, in 2001 and 2005, respectively.

From January to May 2006, she was a Postdoctoral Research Associate with the Department of Electrical and Computer Engineering and the Institute for Systems Research, University of Maryland. She is currently a Wireless Communications Specialist with Meteor Communications Corporation, Kent, WA, working on the research and development of wireless communications technology. Her research interests span a broad range of areas from signal processing to wireless communications and networking, including space-time coding for multiantenna communications, cross-layer design for wireless networks, communications in mobile ad hoc networks and wireless sensor networks, orthogonal frequency division multiplexing systems, ultrawideband communications, and cognitive radio.

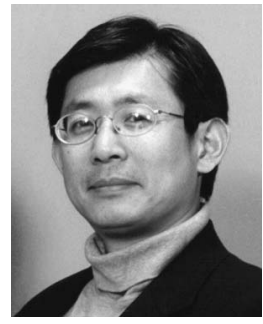

K. J. Ray Liu (F'03) received the B.S. degree from the National Taiwan University, Taipei, Taiwan, in 1983 and the Ph.D. degree from the University of California, Los Angles, in 1990.

$\mathrm{He}$ is a Distinguished Scholar-Teacher with the University of Maryland, College Park. He leads the Maryland Signals and Information Group, conducting research encompassing broad aspects of information technology, including communications and networking, information forensics and security, multimedia signal processing, biomedical imaging, and bioinformatics. He has authored several books, including Cooperative Communications and Networking (Cambridge Univ. Press, 2008), Resource Allocation for Wireless Networks: Basics, Techniques, and Applications (Cambridge Univ. Press, 2008), Ultra-Wideband Communication Systems: The Multiband OFDM Approach (IEEE-Wiley, 2007), Network-Aware Security for Group Communications (Springer, 2007), and Multimedia Fingerprinting Forensics for Traitor Tracing (Hindawi, 2005).

Dr. Liu was a recipient of numerous honors and awards, including Best Paper Awards from the IEEE Signal Processing Society (twice), the IEEE Vehicular Technology Society, and EURASIP. He has been an IEEE Signal Processing Society Distinguished Lecturer and has received the EURASIP Meritorious Service Award and the National Science Foundation Young Investigator Award. He was also a recipient of various teaching and research recognitions from the University of Maryland, including the university-level Invention of the Year Award, the Outstanding Research Award, and the Poole and Kent Senior Faculty Teaching Award from the A. James Clark School of Engineering Faculty. He is the Vice President for Publications and is on the Board of Governors of the IEEE Signal Processing Society. He was the Editor-in-Chief of the IEEE Signal Processing Magazine and the founding Editor-in-Chief of the EURASIP Journal on Applied Signal Processing. 